\section{Closure with an over-the-scope clip allows therapeutic ERCP to be safely performed after acute duodenal perforation during diagnostic endoscopic ultrasound}
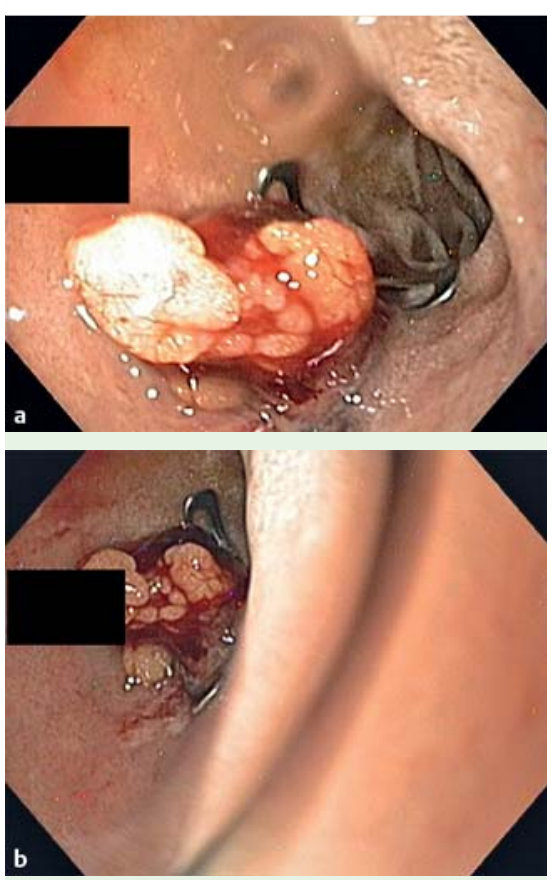

Fig. 1 Endoscopic views showing the overthe-scope clip (OTSC) following its release, successfully closing the perforation with aspirated greater omentum: a patient \#1; b patient \#2.

The use of endoscopic ultrasound (EUS) before endoscopic retrograde cholangiography (ERC), if performed, can prevent two-thirds of unnecessary ERC procedures [1]. Duodenal perforation during diagnostic EUS occurs with an incidence of less than $1 \%$ [2]. When perforation does occur, surgical or conservative management is possible. Surgery permits simultaneous treatment of the perforation and any biliary pathology if present [3]. Conservative management has been described using closure by endoscopic clipping [4]. Endoscopic retrograde cholangiopancreatography (ERCP) is then postponed, so avoiding duodenal maneuver and insufflation that could damage endoscopically placed sutures or aggravate the clinical picture. The over-the-scope clip (OTSC; Ovesco Endosintestinal perforation [5]. that occurred during EUS. vention.
In conclusion, an OTSC is a reliable device in the treatment of acute accidental gastrointestinal perforation during EUS, which allows therapeutic ERC to be performed immediately afterwards.

Endoscopy_UCTN_Code_CPL_1AK_2AB

Competing interests: None

copy $\mathrm{GmbH}$, Tübingen, Germany) is a new full-thickness clipping device that has been used successfully to treat gastro-

We report, to our knowledge, the first two patients to undergo successful therapeutic ERC following treatment with an OTSC for acute duodenal perforation that occurred during diagnostic EUS.

The two women, aged 88 and 67, both presented with cholangitis. In the first patient the perforation occurred at the level of the superior duodenal flexure, after a diagnosis of bile duct lithiasis had been made by EUS. In the second patient the greater omentum was visualized immediately after the pylorus because of a perforation of the superior wall of the bulb

Both perforations were immediately recognized, but we continued to use roomair insufflation - the use of carbon dioxide in this situation is highly recommended. An $11 \mathrm{t} / 6-\mathrm{mm}$ OTSC was released $(\bullet$ Fig. 1), drawing together the margins of the perforation and sealing the wound with aspirated greater omentum. In both patients a small pneumoperitoneum was detected during the procedure ( $\bullet$ Fig. 2 a); however, contrast medium was injected under pressure and no leakage was detected $(\bullet$ Fig. $\mathbf{2 b}$ ).

In each case after the perforation had been successfully closed, endoscopic sphincterotomy was performed to re-establish biliary drainage ( $\bullet$ Fig.2c). Prophylactic antibiotics were started and both patients were kept fasting for 48 hours. Neither patient needed to be managed in the intensive care unit and no further imaging investigations were required. Both patients made good progress, with regression of their inflammatory syndromes, and were discharged from hospital free of symptoms 72 hours after inter-

\section{Gianfranco Donatelli ${ }^{1}$, Bertrand M. Vergeau', Stavros Dritsas ${ }^{2}$, Jean-Loup Dumont ${ }^{1}$, Thierry Tuszynski ${ }^{1}$, Bruno Meduri ${ }^{1}$}

${ }^{1}$ Therapeutic Endoscopy Unit, Hôpital

Privé des Peupliers, Paris, France

2 Department of Hepatology, Hôpital Universitaire Jean Minjoz, Besançon, France

\section{References}

1 Petrov MS, Savides TJ. Systematic review of endoscopic ultrasonography versus endoscopic retrograde cholangiopancreatography for suspected choledocholithiasis. $\mathrm{Br} \mathrm{J}$ Surg 2009; 96: 967-974

2 Jenssen C, Alvarez-Sánchez MV, Napoléon B et al. Diagnostic endoscopic ultrasonography: assessment of safety and prevention of complications. World J Gastroenterol 2012; 18: 4659-4676

3 Leroy J, Donatelli GF, Paveliu S et al. Laparoscopic management of duodenal perforation during endoscopic sphincterotomy for CBD stone. Available from: http://www.websurg. $\mathrm{com} /$ video/?doi=vd01 en3049\&redim=1, Accessed: 2 September 2013

4 Seibert G. Use of an endoscopic clipping device to repair a duodenal perforation. Endoscopy 2003; 35: 189

5 Donatelli G, Leblanc S, Vienne A et al. Is overthe-scope clip a permanently implanted device? Outcome and follow up of clip delivery for fistulas, perforations and bleeding. Gastrointest Endosc 2013; 77: AB 207-AB208

\section{Bibliography}

DOI http://dx.doi.org/

10.1055/s-0033-1344872

Endoscopy 2013; 45: E392-E393

(c) Georg Thieme Verlag KG

Stuttgart · New York

ISSN 0013-726X

\section{Corresponding author}

\section{Gianfranco Donatelli, MD}

Unité d'Endoscopie Thérapeutique

Hôpital Privé des Peupliers

Paris

France

Fax: +33-1-44165615

donatelligianfranco@gmail.com 

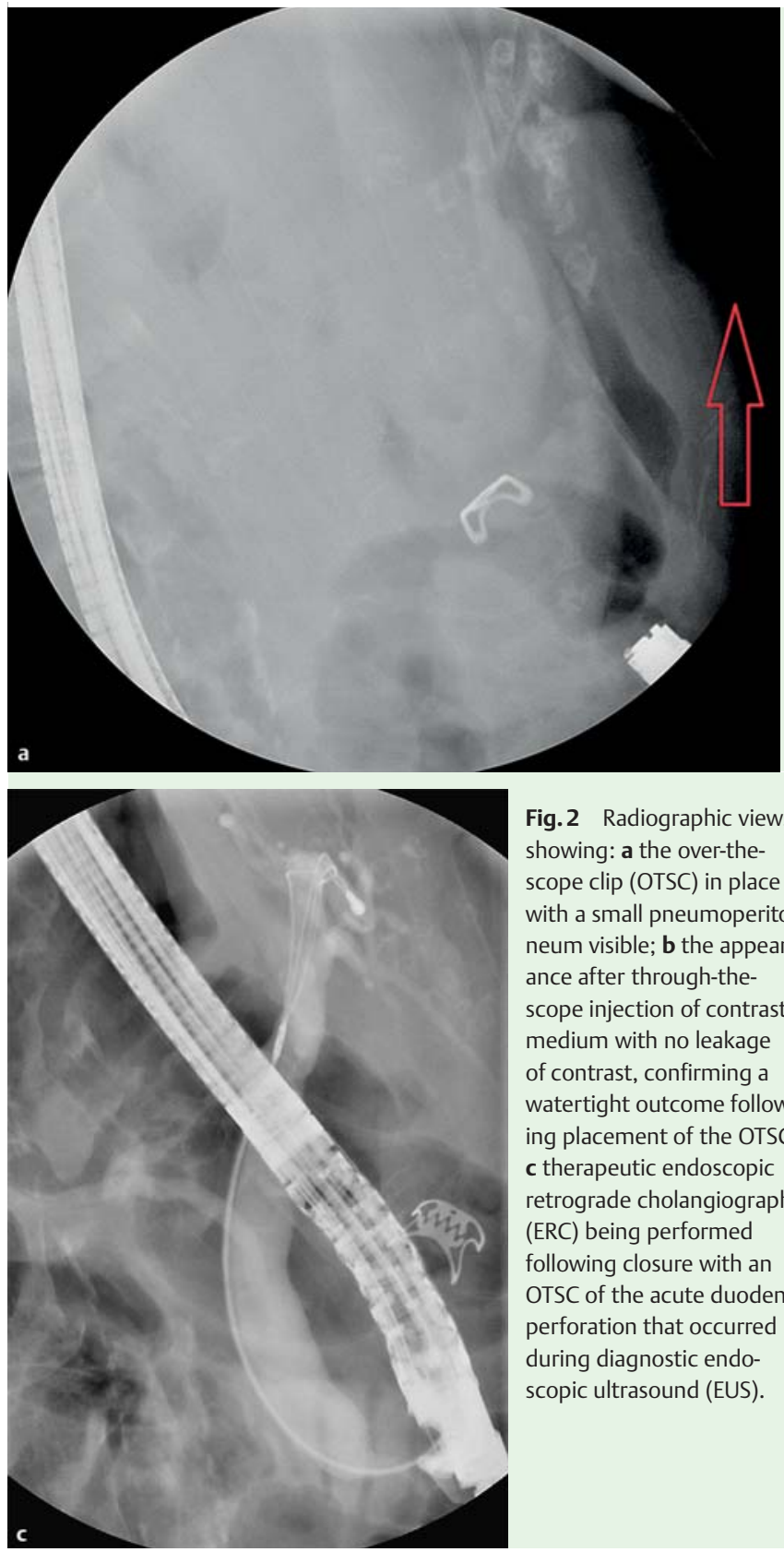
showing: a the over-thescope clip (OTSC) in place ance after through-themedium with no leakage of contrast, confirming a c therapeutic endoscopic (ERC) being performed following closure with an perforation that occurred during diagnostic endoscopic ultrasound (EUS).

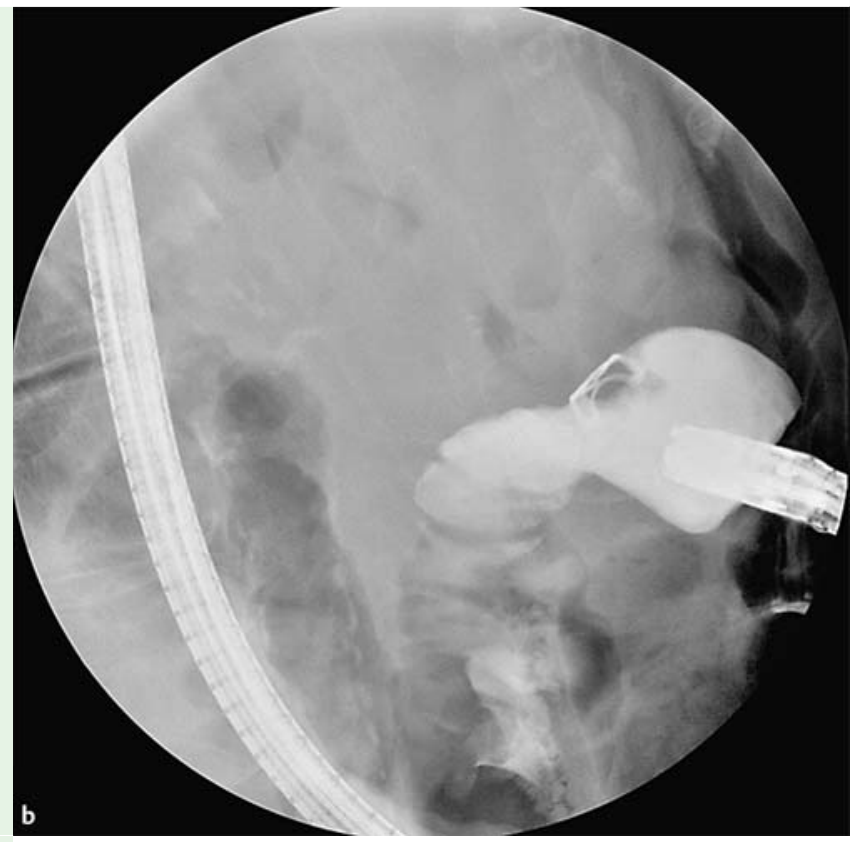

Fig. 2 Radiographic views with a small pneumoperitoneum visible; $\mathbf{b}$ the appearscope injection of contrast watertight outcome following placement of the OTSC; retrograde cholangiography OTSC of the acute duodenal 